RESEARCH ARTICLE

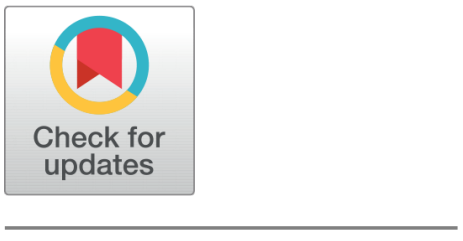

OPEN ACCESS

Received: 06.02.2021

Accepted: 02.05.2021

Published: 12.05 .2021

Citation: Appidi L, Malga BS, Matta S, Pramod Kumar P (2021)

Effects of Thermal Radiation on Temperature and Concentration on MHD Free Convection Flow Past a Vertical Porous Plate in the Presence of Chemical Reaction and Heat Source Parameter. Indian Journal of Science and Technology 14(17): 1354-1363. https://doi.org/ 10.17485/IJST/v14i17.235

* Corresponding author.

appidilakshmi1984@gmail.com

Funding: None

Competing Interests: None

Copyright: (c) 2021 Appidi et al. This is an open access article distributed under the terms of the Creative Commons Attribution License, which permits unrestricted use, distribution, and reproduction in any medium, provided the original author and source are credited.

Published By Indian Society for Education and Environment (iSee)

ISSN

Print: 0974-6846

Electronic: 0974-5645

\section{Effects of Thermal Radiation on Temperature and Concentration on MHD Free Convection Flow Past a Vertical Porous Plate in the Presence of Chemical Reaction and Heat Source Parameter}

\author{
Lakshmi Appidi ${ }^{1,2 *}$, Bala Siddulu Malga ${ }^{2}$, Sweta Matta ${ }^{1,2}$, P Pramod Kumar ${ }^{2,3}$ \\ 1 Department of Mathematics, CMR Technical Campus, Kandlakoya, 501401, Telangana, India \\ 2 Department of Mathematics, GITAM University, Hyderbad, 502329, Telanagana, India \\ 3 Department of Mathematics, B V Raju Institute of Technology, Narsapur, 502313, \\ Telangana, India
}

\section{Abstract}

Objective: To study the effects of chemical reaction and heat source parameter on MHD Free Convection Flow Past a Vertical Porous Plate. Method: The problem is represented by coupled non-direct fractional differential conditions. The non-dimensional conditions of the issue have been tackled mathematically utilizing the Finite-Element strategy. Findings: The effects of governing parameters such as chemical reaction, heat source, permeability, radiation and many others are discussed quantitatively with the help of graphs in these flow fields like velocity, temperature, concentration with the help of skin friction, Nusselt number and Sherwood number. Novelty: The accurateness of the solution to the problem was verified by comparing it with the published previously. The compatibility between the results was found to be high. To strengthen the validity of the numerical results were presented in this work.

Keywords: Temparature; Concentration; Chemical reaction; Mass diffusion; Eckert numeral; Radiation; MHD; Time dependent variable

\section{Introduction}

Free convection stream connecting to heat transference happens commonly in an atmosphere where transformations between the land and air temperature can give escalation to intricated flow influence. The theme of MHD has involved the concentration of huge people of research learners because of its varied exertion. The results of the magnetic arena allowed temperature changes of a stream to play a vital role in ionized gasses, liquid metals and electrolytes. Now the present scholars are interested to know the consequences of the magnetic arena on the temperature dispersal and heat transference when the liquid is not just an electric conductor when it is proficient in producing and gripping convector heat. Heat transmission through induction heat is a great reputation when there are considerations of space submissions, experienced 
temperatures and power engineering.

An unstable magnetohydrodynamics convector heat transmission former a semi-infinite perpendicular permeable stirring dish with inconstant force deliberated by $\mathrm{Kim}^{(1)} \mathrm{Chen}^{(2)}$ talk about mass \& heat allocation in magnetohydrodynamics flow through usual convection as of a porous disposed surface thru variable fence concentration and temperature. Abbas et al. ${ }^{(3)}$ explored radioactivity possessions on magnetohydrodynamics stream in a spongy space. Hossain et al. ${ }^{(4)}$ analysed the consequence of radioactivity on unrestricted convection as of an impermeable perpendicular plate. Hossain and Alim ${ }^{(5)}$ examined, usual radiation-convection collaboration on boundary film flow by the side of a narrow upright cylinder. Hossain et al. ${ }^{(6)}$ deliberated the consequence of radioactivity on allowed convection stream of liquid with inconstant thickness from an absorbent perpendicular plate. Kumar et al. ${ }^{(7)}$ studied the effect of viscous dissipation on the shaky polar liquid of free convection stream of warmth and mass exchange past a vertical semi-endless plate moving in permeable medium, inside the presence of cross over attractive field is examined. Kandasamy et al. ${ }^{(8)}$ analysed the possessions of chemical reaction, mass and heat transmission along with a piece through concentration and heat source with the existence of pressure. Anjalidevi et al. explored $^{(9)}$ the results of a chemical response, mass and heat transference on smooth liquid flow along with a semi-infinite parallel dish. Kumar et al. ${ }^{(10)}$ deliberated contemplate the progression of insecure free convection and move of mass going through an endless vertical permeable plate with a synthetic response by considering pull boundary and gooey dissemination if the plate moves in its plane. The theme of nonlinear radiation and blended convection of the MHD warmth and mass exchange of Maxwell nano liquid stream in permeable media with Arrhenius dynamic response is inspected by Salawu et al. ${ }^{(11)}$ Salahuddin et al. ${ }^{(12)}$ initiated MHD sway on dramatically differing consistency of Williamson liquid stream with variable conductivity and diffusivity. Expects to consider the impact of Heat source on an MHD Casson liquid through a vertical fluctuating permeable plate by Goud et al. ${ }^{(13)}$ Makinde et al. ${ }^{(14)}$ scrutinized unconfined convection stream through thermal radioactivity and mass conversion past a moving erect permeable platter. Ravikumar et al ${ }^{(15)}$ has examined magnetohydrodynamic couple diffusivity with chemical sensible stream moving overall double standing plates of a porous channel. The result of inconstant pressure and temperature in company with merged results convective Rivlin-Ericksen flow past by way of a semi-infinite vertical penetrable plate was deliberated by Ravikumar et $\mathrm{al}^{(16)}$. Effect of organic impact on hot spell and mass transmission of a marginal sheet flow-thru heat swapping was explored by Chamkha ${ }^{(17)}$ Raju et $\mathrm{al}^{(18)}$ deliberated magnetohydrodynamic convective flow in a parallel panel inside the presence of viscous degeneracy in cooperation with joule heating thru a penetrable channel. Malga et $\mathrm{al}^{(19)}$ look over the conclusion of burning liquefied on unrestricted convector heat transmission over a spongy channel in the existence of induced magnetic arena. Kumar et al. ${ }^{(20)}$ analysed the result of Soret numeral on magnetohydrodynamic allowed convection movement of hot spell and mass transmission of an electrically leading non-Newtonian liquid through an erect working on a porous plate. Poddar et al. ${ }^{(21)}$ directed on the magneto-hydrodynamics (MHD) limit layer (BL) warmth and mass exchange stream of thermally transmitting and dissipative liquid over a boundless plate of vertical direction with the contribution of prompted attractive field and warm dispersion Appidi et al. ${ }^{(2)}$ analysed one-dimensional instable unrestricted convector heat and mass transmission flow of micro-polar fluid surrounded in a permeable panel thru a vicious circle. Vijayaraghavan and Karthikeyan ${ }^{(23)}$ explored the effects of heat and mass transfer in MHD flow of a Casson fluid involved a moving vertical porous plate. Omamoke et al. ${ }^{(24)}$ investigated radiation, thick scattering and warmth source consequences for magneto-hydrodynamic free convection stream, of a gooey incompressible liquid over a slanted permeable plate. The extant study is also besides with Eckert number extension of Effects of MHD Free Convection Flow Past a Vertical Porous Plate in the Presence of Thermal Radiation and Chemical Reaction.

The objective of the present work is to study the effects of thermal radiation on temperature and concentration on MHD free convection flow past a vertical porous plate in the presence of chemical reaction and heat source parameter. It has been observed that the results of thermal radiation, chemical reaction, heat source and other parameters effects shown graphically and the solution of the problem by using the Galkerin-Finite element method. The current research is an extension of the study done by Umamaheswar et al. ${ }^{(25)}$ with the addition of Eckert number in the temperature field. The accuracy of the present research has been verified by comparing it with the previous results of. Both previous and present consequences on velocity, temperature and concentration fields are coinciding and showing similar results.

\section{Problem Formulation:}

The instable stream of an electrically accompanying incompressible, viscid, radiative along with chemically reactive liquid past an immeasurable erect plate with inconstant temperature and concentration over an absorbent technique in the existence of temperature basis has been measured. The stream is supposed to be in $\mathrm{X}^{\star}$-a path that is engaged along with the vertical plate in the upward direction. The $\mathrm{Y}^{*}$-direction is taken to be normal to the plate. Originally, it is supposed that together liquefied and plates are on respite and at similar temperature $T_{\infty}^{*}$ and concentration $C_{\infty}^{*}$. At $t^{*}>0$ the temparature and concentration of 
the plate $\mathrm{Y}^{*}=0$ is raised to $T_{\infty}^{*}+\left(T_{w}^{*}-T_{\infty}^{*}\right) e^{a^{*} t^{*}}$ and $C_{\infty}^{*}+\left(C_{w}^{*}-C_{\infty}^{*}\right) e^{a^{*} t^{*}}$ with time $\mathrm{t}$ and there after remains constant that of $y^{*} \rightarrow \infty$ is lowered to $T_{\infty}^{*}$ and $C_{\infty}^{*}$. A diagonal magnetic field of an unvarying asset is supposed to be functionalized as usual to a plate. The persuaded viscous dissipation and a magnetic field are supposed to be insignificant as the magnetic Reynold numeral of the stream is considered to be very less. The divergence effects are supposed to be small and hence the electric arena is too small. The absorption of the diffusing classes in the double mix is supposed to be negligible in contrast with other chemical reactions, which are contemporary and henceforth Dufour and Soret results are insignificant. Now the hall effect of magnetohydrodynamic and magnetic degeneracy is deserted.

As per the previous supposition by the normal Boussineq's calculation, the leading equivalences and borderline circumstances were mentioned as below

Momentum:

$$
\frac{\partial u^{*}}{\partial t^{*}}=v \frac{\partial^{2} u^{*}}{\partial y^{* 2}}+\beta\left(T^{*}-T_{\infty}^{*}\right)+\beta^{*}\left(C^{*}-C_{\infty}^{*}\right)-\frac{\sigma B_{0}^{2}}{\rho} u^{*}-\frac{v}{K_{p}^{*}} u^{*}
$$

Energy:

$$
\rho C_{p} \frac{\partial T^{*}}{\partial t^{*}}=k \frac{\partial^{2} T^{*}}{\partial y^{* 2}}+Q_{0}\left(T^{*}-T_{\infty}^{*}\right)-\frac{\partial q_{r}^{*}}{\partial y^{*}}+\frac{v}{C_{p}}\left(\frac{\partial u^{*}}{\partial y^{*}}\right)^{2}
$$

Concentration:

$$
\frac{\partial C^{*}}{\partial t^{*}}=D \frac{\partial^{2} C^{*}}{\partial y^{* 2}}-K_{r}^{*}\left(C^{*}-C_{\infty}^{*}\right)
$$

Initial and Boundary conditions are:

$$
\begin{aligned}
& u^{*}=0, T^{*}=T_{\infty}^{*}, C^{*}=C_{\infty}^{*} \text { forall } y^{*}, t^{*} \leq 0 \\
& t^{*}>0: u^{*}=u_{0}, T^{*}=T_{\infty}^{*}+\left(T_{w}^{*}-T_{\infty}^{*}\right) e^{a^{*} t^{*}}, C^{*}=C_{\infty}^{*}+\left(C_{w}^{*}-C_{\infty}^{*}\right) e^{a^{*} t^{*}} \text { at } y^{*}=0 \\
& u^{*} \rightarrow 0, T^{*} \rightarrow T_{\infty}, C^{*} \rightarrow C_{\infty} \text { as } y^{*} \rightarrow \infty
\end{aligned}
$$

The resident glowing interest in the instance of an ocular tinny dull vapour is stated as

$$
\frac{\partial q_{r}^{*}}{\partial y^{*}}=4 a^{\prime} \sigma^{*}\left(T_{\infty}^{*^{4}}-T^{*^{4}}\right)
$$

Here $a^{\prime}$ denotes average absorption co-efficient and $\sigma^{*}$ denotes Stefan-Boltzmann persistent. Now we done transformations inside of the flow as adequately slight so as to $\mathrm{T}^{*}$ can be states as a linear occupation of $T^{*}$ after that follows Taylor's procedure to enlarge $\mathrm{T}^{*}$ around the unrestricted flow temparature $T_{\infty}^{*^{4}}$ and ignoring the greater level terms. This development is in the succeeding calculation : $\mathrm{T}^{*} \cong 4 T_{\infty}^{*^{3}}-3 T_{\infty}^{*^{4}}$.

Now equation(2.2) gives as follows

$$
\rho C_{p} \frac{\partial T^{*}}{\partial t^{*}}=k \frac{\partial^{2} T^{*}}{\partial y^{* 2}}+q_{0}\left(T^{*}-T_{\infty}^{*}\right)-16 a^{\prime} \sigma^{*} T_{\infty}^{*^{3}}\left(T^{*}-T_{\infty}^{*}\right)
$$

Familiarizing the bellowed dimensionless amounts:

$$
\begin{aligned}
& \mathrm{u}=\frac{u^{*}}{u_{0}}, \mathrm{t}=\frac{t^{*} u_{0}^{2}}{v}, \mathrm{y}=\frac{y^{*} u_{0}}{v}, \theta=\frac{T^{*}-T_{\infty}^{*}}{T_{w}^{*}-T_{\infty}^{*}}, \mathrm{c}=\frac{C^{*}-C_{\infty}^{*}}{C_{w}^{*}-C_{\infty}^{*}}, \mathrm{Gr}=\frac{v^{\mathrm{g}} \beta\left(T_{W}^{*}-T_{\infty}^{*}\right)}{u_{0}^{3}}, \mathrm{Gc}=\frac{v^{\mathrm{g}} \beta^{*}\left(C_{W}^{*}-C_{\infty}^{*}\right)}{u_{0}^{3}}, \mathrm{M}=\frac{\sigma v B_{0}^{2}}{\rho u_{0}^{2}}, \mathrm{~K}=\frac{K_{p}^{*} u_{0}^{2}}{v^{2}}, \\
& P_{r}=\frac{\mu C_{p}}{k}, \mathrm{R}=\left(\frac{16 a^{\prime} \sigma^{*} T_{\infty}^{* 3} v^{2}}{k u_{0}^{2}}\right)_{\mu} S c=\frac{v}{D}, K_{r}=\frac{K_{r}^{*} v}{u_{0}^{2}}, \mathrm{a}=\frac{a^{*} v}{u_{0}^{2}} \\
& Q=\frac{Q_{0} v^{2}}{k u_{0}^{2}}, E c=\frac{u_{0}^{2}}{C_{p}\left(T_{w}^{*}-T_{\infty}^{*}\right)}
\end{aligned}
$$


The dimensionless parameters mentioned as modified Grashof number-(Gr), permeability parameter-(K), Grashof number(Gc), Eckert number-(Ec), radiation parameter-(R), heat source parameter-(Q), Prandtl number-(Pr), Schmidt number-(Sc), magnetic parameter- $(\mathrm{M})$ and chemical reaction parameter- $(\mathrm{Kr})$. After familiarizing the dimensionless amounts in to the equivalences (2.1),(2.2)and (2.3), these equivalences changed as follows

$$
\begin{gathered}
\frac{\partial u}{\partial t}=\frac{\partial^{2} u}{\partial y^{2}}+G_{r} \theta+G_{m} C-M u-\frac{1}{K} u \\
P_{r} \frac{\partial \theta}{\partial t}=\frac{\partial^{2} \theta}{\partial y^{2}}+Q \theta-R \theta+E c\left(\frac{\partial u}{\partial y}\right)^{2} \\
\frac{\partial C}{\partial t}=\frac{1}{S c} \frac{\partial^{2} C}{\partial y^{2}}-K_{r} C
\end{gathered}
$$

The conforming boundary \& initial surroundings are

$$
\begin{aligned}
& u=0, \theta=0, C=0 \quad \text { forall } y, t \leq 0 \\
& t>0: u=1, \theta=e^{a t}, C=e^{a t} \text { at } y=0 \\
& u \rightarrow 0, \theta \rightarrow 0, C \rightarrow 0 \quad \text { as } y \rightarrow \infty
\end{aligned}
$$

\section{Method of Solution}

Now the instable, nonlinear, doubled PDE. (2.7)-(2.9) together with their boundary conditions (2.10) have been solved logically by using the finite element method as below

parameterization of an area into limited components

Construction of module component equivalences

Uniting component equations

Applying restraint borderline conditions

Concluding the result of gathered equations

Finite element Galerkin procedure is employed to solve the equation (2.7),(2.8), and (2.9) concluded a two-nodded linear element $(\mathrm{e})\left(y_{j} \leq y \leq y_{k}\right)$ is

$$
\begin{gathered}
\int_{y_{j}}^{y_{k}} N^{T}\left[\frac{\partial^{2} u}{\partial y^{2}}-\frac{\partial u}{\partial t}-\left(M+\frac{1}{K}\right) u+G_{r} \theta+G_{c} C\right] d y \\
\int_{y_{j}}^{y_{k}}\left[\frac{\partial N}{\partial y} \cdot \frac{\partial u^{(e)}}{\partial y}-N^{T}\left(-\frac{\partial u^{(e)}}{\partial t}-\left(M+\frac{1}{K}\right) u^{(e)}+R\right)\right] d y=0 \\
\text { where } R=\left(G_{r} \theta+G_{c} C\right), N=\left[N_{j}, N_{k}\right], \phi^{(e)}=\left[\begin{array}{c}
u_{j} \\
u_{k}
\end{array}\right], \\
u^{(e)}=N . \phi^{(e)}, \quad N_{j}=\frac{y_{k}-y}{l^{(e)}}, \quad N_{k}=\frac{y-y_{j}}{l^{(e)}}, \quad l^{(e)}=y_{k}-y_{j}=h
\end{gathered}
$$

The element equation is given by

$$
\begin{gathered}
\int_{y_{j}}^{y_{k}}\left[\begin{array}{ll}
N_{j}^{\prime} N_{j}^{\prime} & N_{j}^{\prime} N_{k}^{\prime} \\
N_{k}^{\prime} N_{j}^{\prime} & N_{k}^{\prime} N_{k}^{\prime}
\end{array}\right]\left[\begin{array}{c}
u_{j} \\
u_{k}
\end{array}\right] d y+\left[\begin{array}{ll}
N_{j} N_{j} & N_{j} N_{k} \\
N_{k} N_{j} & N_{k} N_{k}
\end{array}\right]\left[\begin{array}{c}
\dot{u}_{J} \\
\dot{u}_{k}
\end{array}\right] d y+ \\
\left(M+\frac{1}{K}\right)\left[\begin{array}{cc}
N_{j} N_{j} & N_{j} N_{k} \\
N_{k} N_{j} & N_{k} N_{k}
\end{array}\right]\left[\begin{array}{c}
u_{j} \\
u_{k}
\end{array}\right] d y-R\left[\begin{array}{c}
N_{j} \\
N_{k}
\end{array}\right] d y=0 \\
\frac{1}{l^{(e)}}\left[\begin{array}{cc}
1 & -1 \\
-1 & 1
\end{array}\right]\left[\begin{array}{c}
u_{j} \\
u_{k}
\end{array}\right]+\left(M+\frac{1}{K}\right) \frac{l^{(e)}}{6}\left[\begin{array}{ll}
2 & 1 \\
1 & 2
\end{array}\right]\left[\begin{array}{c}
u_{j} \\
u_{k}
\end{array}\right]+\frac{l^{(e)}}{6}\left[\begin{array}{ll}
2 & 1 \\
1 & 2
\end{array}\right]\left[\begin{array}{c}
\dot{u} \\
\dot{u_{k}}
\end{array}\right] d y-R \frac{l^{(e)}}{2}\left[\begin{array}{l}
1 \\
1
\end{array}\right]=0
\end{gathered}
$$

where prime and dot referrers differentiation with respect to $\mathrm{y}$ and $\mathrm{t}$ respectively, accumulating the element equation intended for dual successive elements $\left(y_{i-1} \leq y \leq y_{i}\right)$ and $\left(y_{i} \leq y \leq y_{i+1)}\right)$, the following is obtained.

$$
\frac{1}{l^{(e)}}\left[\begin{array}{ccc}
1 & -1 & 0 \\
-1 & 2 & -1 \\
0 & -1 & 1
\end{array}\right]\left[\begin{array}{c}
u_{i-1} \\
u_{i} \\
u_{i+1}
\end{array}\right]+\left(M+\frac{1}{K}\right) \frac{l^{(e)}}{6}\left[\begin{array}{ccc}
2 & 1 & 0 \\
1 & 4 & 1 \\
0 & 1 & 2
\end{array}\right]\left[\begin{array}{c}
u_{i-1} \\
u_{i} \\
u_{i+1}
\end{array}\right]+\frac{l^{(e)}}{6}\left[\begin{array}{ccc}
2 & 1 & 0 \\
1 & 4 & 1 \\
0 & 1 & 2
\end{array}\right]\left[\begin{array}{c}
\dot{u}_{i-1} \\
\dot{u}_{i} \\
\dot{u}_{i+1}
\end{array}\right]=R \frac{l^{(e)}}{2}\left[\begin{array}{c}
1 \\
2 \\
1
\end{array}\right]
$$


Here and now take the place of row conforming to the node i to null from(14) the variance schemes with $l^{(e)}=h$ is

$$
\frac{1}{h^{2}}\left(-u_{i-1}+2 u_{i}-u_{i+1}\right)+\left(M+\frac{1}{K}\right) \frac{1}{6}\left(u_{i-1}+4 u_{i}+u_{i+1}\right)+\frac{1}{6}\left(\dot{u}_{i-1}+4 \dot{u}_{i}+\dot{u}_{i+1}\right)=R
$$

By employing trapezoidal rule, bellowed structure of calculations in crank -Nicolson to the equation (16), we acquire:

$$
A_{1} u_{i-1}^{n+1}+A_{2} u_{i}^{n+1}+A_{3} u_{i+1}^{n+1}=A_{4} u_{i-1}^{n}+A_{5} u_{i}^{n}+A_{6} u_{i+1}^{n}+12 k R
$$

where, $R=G r T_{i}^{j}+G c C_{i}^{j}$

Similarly, for the equation (10),(11) following equations are obtained:

$$
\begin{gathered}
C_{1} \theta_{i-1}^{n+1}+C_{2} \theta_{i}^{n+1}+C_{3} \theta_{i+1}^{n+1}=C_{4} \theta_{i-1}^{n}+C_{5} \theta_{i}^{n}+C_{6} \theta_{i+1}^{n}+12 k P r \\
D_{1} C_{i-1}^{n+1}+D_{2} C_{i}^{n+1}+D_{3} C_{i+1}^{n+1}=D_{4} C_{i-1}^{n}+D_{5} C_{i}^{n}+D_{6} C_{i+1}^{n} \\
A_{1}=-6 r+N k+2 ; \quad A_{2}=12 r+4 N k+8 ; \\
A_{4}=6 r-N k+2 ; \quad A_{5}=-12 r-4 N k+8 ; \quad A_{6}=6 r-N k+2 ; \\
C_{1}=-6 r+2 P r+R k-Q k ; C_{2}=12 r+8 P r+4 R k-4 Q k ; C_{3}=-6 r+2 P r+R k+Q k \\
C_{4}=6 r+2 P r-R k+Q k ; C_{5}=-12 r+8 P r-4 R k+4 Q k ; \quad C_{6}=6 r+2 P r-R k+Q k ; \\
D_{1}=-6 r+S c K r k+2 S c ; D_{2}=12 r+4 S c K r k+8 S c ; D_{3}=-6 r+S c K r k+2 S c ; \\
D_{4}=6 r-S c K r k+2 S c ; D_{5}=-12 r-4 K r S c k+8 S c ; D_{6}=6 r-S c K r k+2 S c .
\end{gathered}
$$

Here $R^{* *}=E c\left(\frac{\partial u}{\partial y}\right)^{2}$ and $r=\frac{k}{h^{2}}$ where $\mathrm{k}, \mathrm{h}$ represents mesh magnitude through $\mathrm{y}$ and time direction respectively. The mesh scheme contains $\mathrm{h}=0.1$ for velocity, concentration contours here $\mathrm{k}=0.001$ has been measured for the result of reckoning the equivalences (3.7 - 3.9), enchanting $\mathrm{i}=1(1) \mathrm{n}$ and considering boundary initial conditions (2.4) and (2.10) the mentioned below equations we get.

$A_{i} X_{i}=B_{i}$, where $i=1,2,3 \ldots$

In the above equation $A_{i}$ 's ,B's and X's are matrices of order with n-elements. Thomas procedure was used to arrive at the determination of the previous equivalences. The concentration, velocity and temperature also the mathematical solutions of the equivalences are derived through c-programme in direction to show the stability and also the convergence of finite element Galerkin procedure. A similar method was followed with a small change in worth's of $\mathrm{h}$ and $\mathrm{k}$ and it was detected that no substantial deviations occurred in the worth's of $\mathrm{u}, \theta$, and $\mathrm{C}$. Thus, it can be inferred that the finite element Galerkin procedure is steady and convergent.

Now the non-dimensional quantities of Sherwood number, Nusselt number and Skin friction are shown below

\begin{tabular}{llllllllll}
\multicolumn{10}{c}{ Table 1. $\tau=\left(\frac{\partial u}{\partial y}\right)$ at $y=0$} \\
\hline $\mathrm{M}$ & $\mathrm{Gr}$ & $\mathrm{Gc}$ & $\mathrm{K}$ & $\mathrm{Sc}$ & $\operatorname{Pr}$ & $\mathrm{Kr}$ & $\mathrm{Q}$ & $\mathrm{R}$ & $\tau$ \\
\hline 0.2 & 0.2 & 0.2 & 0.1 & 0.22 & 0.71 & 0.9 & 0.7 & 0.8 & 0.50751758 \\
0.23 & 0.2 & 0.2 & 0.15 & 0.96 & 0.71 & 0.9 & 0.7 & 0.8 & 0.42229164 \\
0.3 & 0.5 & 0.5 & 0.23 & 0.78 & 0.71 & 0.9 & 0.7 & 0.8 & 0.27157485 \\
0.3 & 0.3 & 0.3 & 0.27 & 0.78 & 0.71 & 0.9 & 0.7 & 0.8 & 0.29112482 \\
0.5 & 0.2 & 0.3 & 0.3 & 0.78 & 0.71 & 0.9 & 0.7 & 0.8 & 0.30960953 \\
0.5 & 0.5 & 0.5 & 0.3 & 0.78 & 0.71 & 0.9 & 0.7 & 0.8 & 0.22847712 \\
0.5 & 0.2 & 0.2 & 0.4 & 0.99 & 0.71 & 0.9 & 0.7 & 0.8 & 0.26683104 \\
\hline
\end{tabular}




\begin{tabular}{llcl}
\multicolumn{4}{c}{ Table 2. $\mathrm{N}_{\mathrm{u}}=-\mu\left(\frac{\partial \theta}{\partial y}\right)$ at $y=0$} \\
\hline $\operatorname{Pr}$ & $\mathrm{R}$ & $\mathrm{Q}$ & $\mathrm{Nu}$ \\
\hline 0.4 & 0.1 & 0.1 & 0.09248495 \\
0.6 & 0.2 & 0.1 & 0.17016983 \\
0.65 & 0.1 & 0.2 & 0.08935261 \\
0.71 & 0.2 & 0.3 & 0.09929514 \\
0.78 & 0.1 & 0.2 & 0.11028004 \\
0.84 & 0.2 & 0.4 & 0.07662249 \\
0.96 & 0.2 & 0.21301460 \\
0.98 & 0.3 & 0.1 & 0.21513414 \\
1.5 & 0.2 & 0.4 & 0.26314926 \\
\hline
\end{tabular}

Table 3. $S h=-\left(\frac{\partial C}{\partial y}\right)$ at $y=0$

\begin{tabular}{lll}
\hline Sc & $\mathrm{Kr}$ & Sh \\
\hline 0.22 & 0.9 & 0.213604 \\
0.60 & 0.9 & 0.3428 \\
0.78 & 0.9 & 0.40619 \\
0.96 & 0.9 & 0.46015 \\
0.22 & 0.1 & 0.06786 \\
0.22 & 0.3 & 0.09135 \\
0.22 & 0.5 & 0.11436 \\
0.22 & 0.7 & 0.13691 \\
0.84 & 0.9 & 0.42507 \\
0.59 & 0.3 & 0.20149 \\
\hline
\end{tabular}

\section{Results and Discussion}

An investigative education has been supported out on the Magnetohydrodynamics flow of a viscous fluid. In the present study possessions of physical constraints such as modified Grashof number-(Gr), permeability parameter-(K), Grashof number$(\mathrm{Gc})$, Eckert number-(Ec), radiation parameter-(R), heat source parameter-(Q), Prandtl number-(Pr), Schmidt number-(Sc), magnetic parameter- $(\mathrm{M})$ and chemical reaction parameter- $(\mathrm{Kr})$ on species concentration, Velocity and Temperature are deliberated thru the graphs were labelled as $1(\mathrm{a})-1(\mathrm{e}) ; 2(\mathrm{a})-2(\mathrm{~d})$ and 3(a)-3(c) respectively.

The report of permeability parameter - K considered on Figure 1 (a) on the field of velocity, as increasing values of permeability velocity also increase.

The effects of Eckert number-Ec on concentration, Temperature and velocity fields are represented in Figure 3(c), Figure 2(d) and Figure 1(e) respectively. As Eckert number raises both temperature and velocity also raised but, in the concentration, field increasing of Eckert number concentration decrease.

In the temperature field radiation parameter-R consequences posted in Figure 2(a). We mentioned my observation is here increasing of radiation temperature decrease.

The observed results of Schmidt number-Sc and Chemical reaction parameter-Kr on in the field of concentration is decreasing parallelly with increasing of Sc, $\mathrm{K}_{r}$ and these are mentioned inFigure 3(a) and Figure 3(b) accordingly.

Modified Grashof number-Gr, Grashof number-Gc results are shown graphically in Figure 1(b) and Figure 1(c) in the field of velocity. We can observe there as increasing of those numbers velocity also raised accordingly.

Figure 1(d) mentioned the effect of the Magnetic parameter- $M$ on the velocity field, it is observed here increasing of $M$ velocity decrease.

The Prandtl number- Pr consequences showcased in Figure 2(c) in the temperature field and observed temperature decreases with an increase of Pr.

Heat Source parameter-Q results noticed in Figure 2(b) in temperature field as temperature raising with raising of $Q$. 

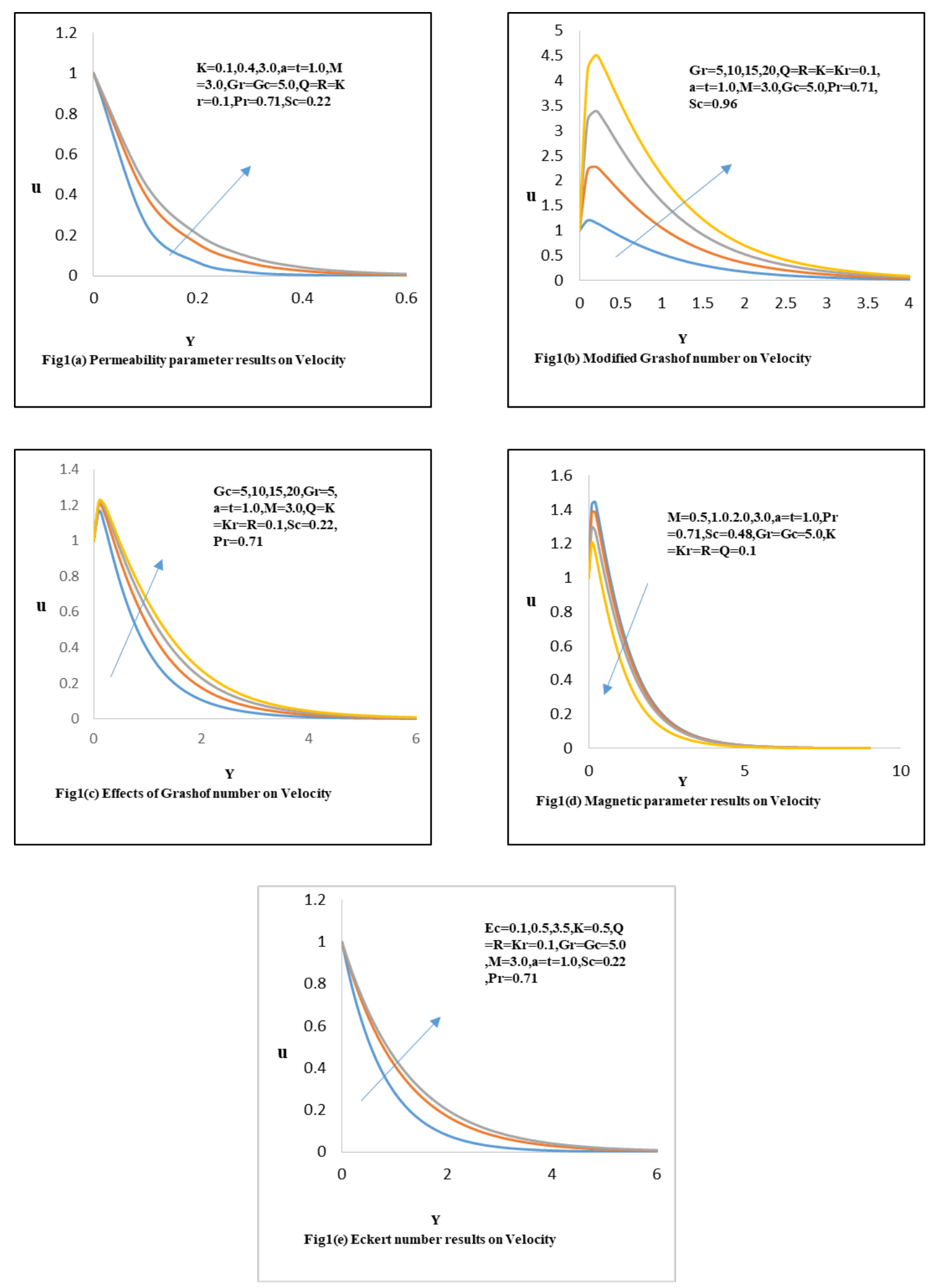

Fig 1. 

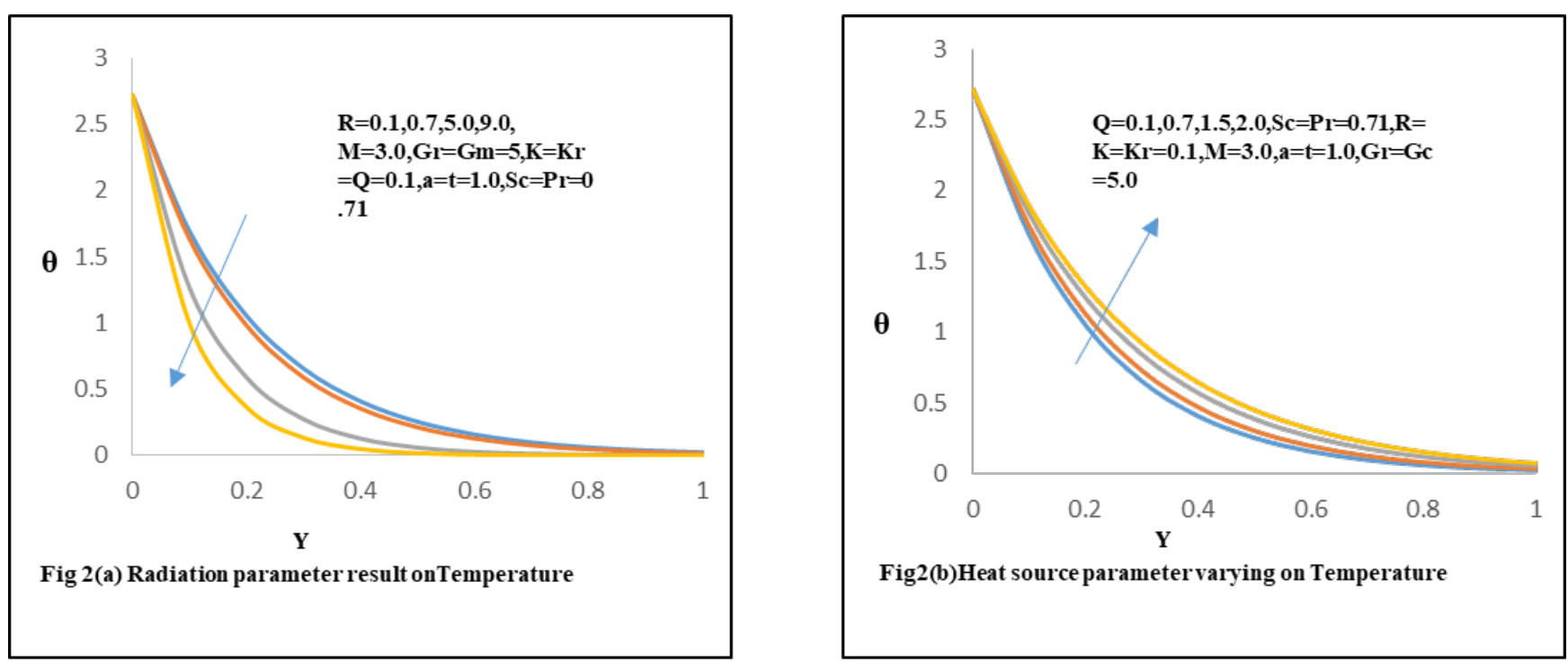

Fig2(b)Heat source parametervarying on Temperature
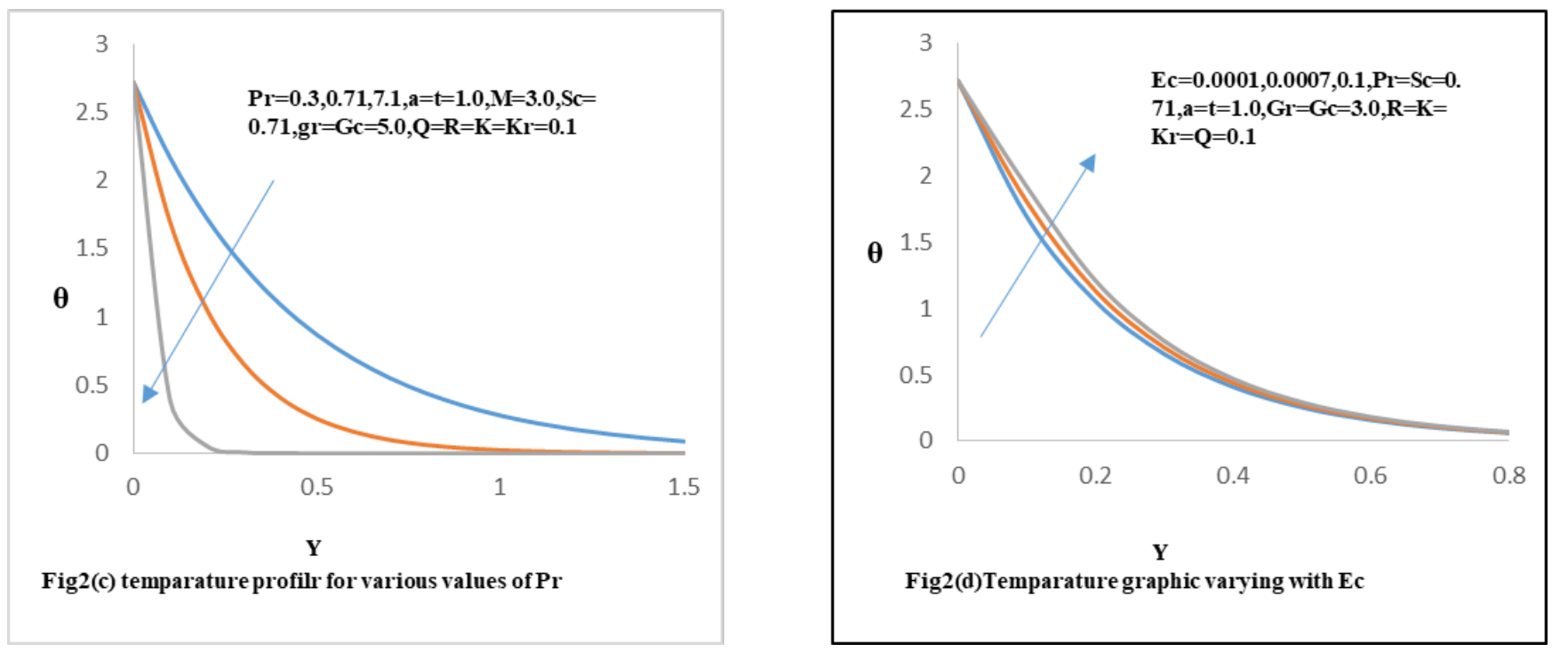

Fig 2. 

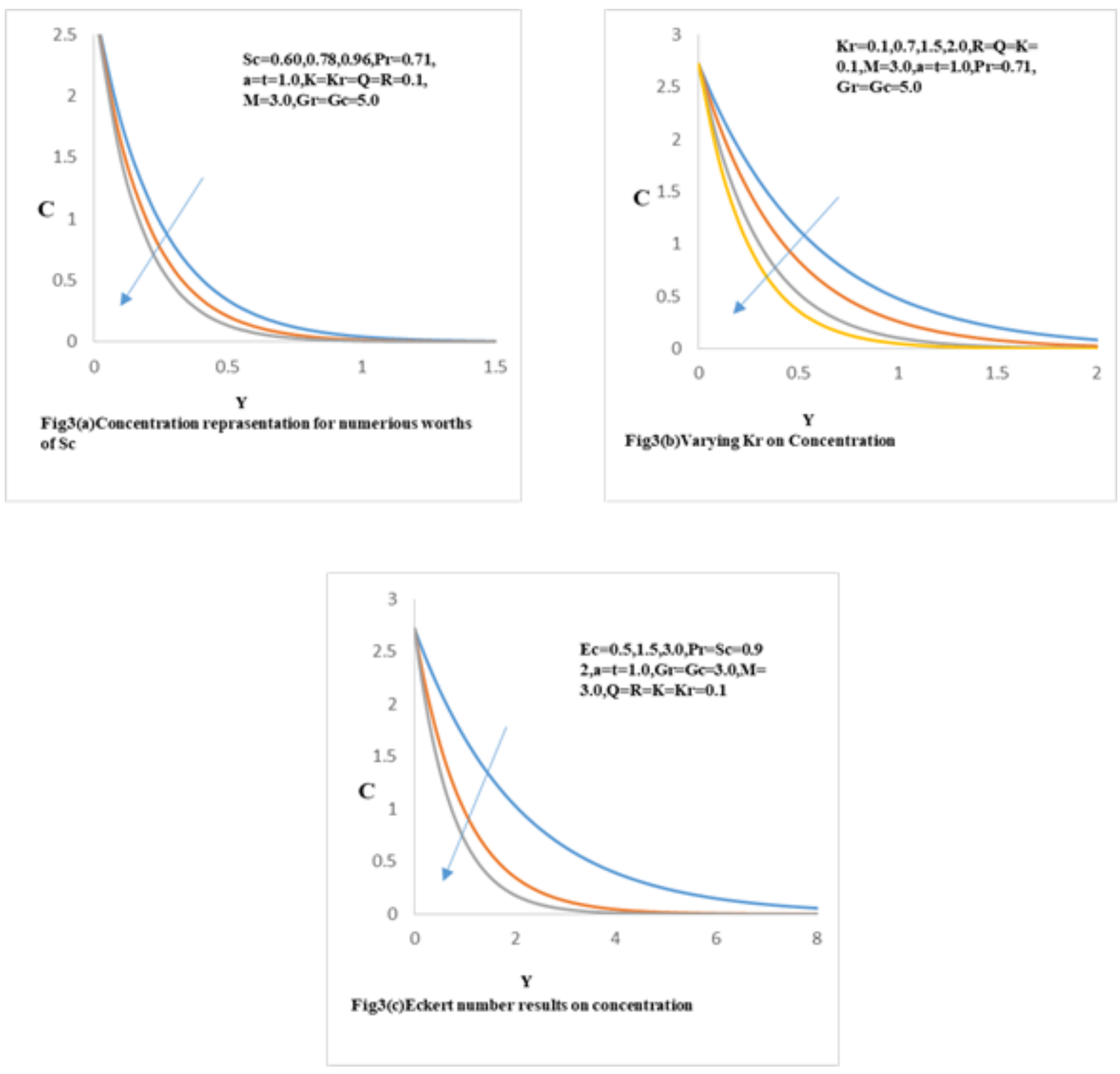

Fig 3.

\section{Conclusions}

The present document concludes as follows :

- Temperature field increases with increasing rate of heat source parameter in the present flow of the fluid .

- Velocity description declines with effect of magnetic constraint in flow fluid.

- The temperature fluid flow at any point of the flow decreases as if there is an increasing of radiation parameter and Prandtl number.

- Concentration profile decreases with increasing of chemical reaction parameter and Schmidth number.

- In the velocity field fluid flow increasing where the permeability parameter, Grashof number and modified Grashof number increases.

- Concentration decreases with effect of increasing Eckert number in the fluid flow. 
- The velocity, temperature rises with the increasing effect of Eckert number

\section{References}

1) Kim YJ. Unsteady MHD convective heat transfer past a semi-infinite vertical porous moving plate with variable suction. International Journal of Engineering Science. 2000;38(8):833-845. Available from: https://dx.doi.org/10.1016/s0020-7225(99)00063-4.

2) Chen $\mathrm{CH}$. Heat and mass transfer in MHD flow by natural convection from a permeable, inclined surface with variable wall temperature and concentration. Acta Mechanica. 2004;172:219-235. Available from: https://doi.org/10.1007/s00707-004-0155-5.

3) Abbas Z, Hayat T. Radiation effects on MHD flow in a porous space. International Journal of Heat and Mass Transfer. 2008;51(5-6):1024-1033. Available from: https://dx.doi.org/10.1016/j.ijheatmasstransfer.2007.05.031.

4) Hossain MA, Alim MA, Rees DAS. The effect of radiation on free convection from a porous vertical plate. International Journal of Heat and Mass Transfer. 1999;42(1):181-191. Available from: https://dx.doi.org/10.1016/s0017-9310(98)00097-0.

5) Hossain MA, Alim MA. Natural convection-radiation interaction on boundary layer flow along a thin vertical cylinder. Heat and Mass Transfer. 1997;32(6):515-520. Available from: https://dx.doi.org/10.1007/s002310050153.

6) Hossain MA, Khanafer K, Vafai K. The effect of radiation on free convection flow of fluid with variable viscosity from a porous vertical plate. International Journal of Thermal Sciences. 2001;40(2):115-124. Available from: https://dx.doi.org/10.1016/s1290-0729(00)01200-x.

7) kumar PP, Malga BS, Matta S, Appidi L. Effect of Viscous Dissipation on Unsteady MHD Free Convection Mass Transfer Flow of Polar Fluid over a Vertical Porous Plate in Presence of Thermal Diffusion and Heat source. International Journal of Advanced Science and Technology. 2020;29(05):1050710519. Available from: http://sersc.org/journals/index.php/IJAST/article/view/24159.

8) Kandasamy R, Periasamy K, Prabhu KKS. Effects of chemical reaction, heat and mass transfer along a wedge with heat source and concentration in the presence of suction or injection. International Journal of Heat and Mass Transfer. 2005;48(7):1388-1394. Available from: https://dx.doi.org/10.1016/j. ijheatmasstransfer.2004.10.008.

9) Anjalidevi SP, Kandasamy R. Effects of chemical reaction, heat and mass transfer on laminar flow along a semi infinite horizontal plate. Heat and Mass Transfer. 1999;35(6):465-467. Available from: https://dx.doi.org/10.1007/s002310050349.

10) Kumar PP, Malga BS, Matta S, Appidi L. Finite element analysis for unsteady free convection and mass transfer flow of a viscous fluid past an accelerated vertical porous plate with suction and chemical reaction. In: and others, editor. AIP Conference Proceedings;vol. 2246. 2020;p. 20028. Available from: https://doi.org/10.1063/5.0014498.

11) Salawu SO, Fatunmbi EO, Okoya SS. MHD heat and mass transport of Maxwell Arrhenius kinetic nanofluid flow over stretching surface with nonlinear variable properties. Results in Chemistry. 2021;3:100125. Available from: https://dx.doi.org/10.1016/j.rechem.2021.100125.

12) Salahuddin T, Khan M, Saeed T, Ibrahim M, Chu YM. Induced MHD impact on exponentially varying viscosity of Williamson fluid flow with variable conductivity and diffusivity. Case Studies in Thermal Engineering. 2021;25:100895. Available from: https://dx.doi.org/10.1016/j.csite.2021.100895.

13) Goud BS, Kumar PP, Malga BS. Effect of Heat source on an unsteady MHD free convection flow of Casson fluid past a vertical oscillating plate in porous medium using finite element analysis. Partial Differential Equations in Applied Mathematics. 2020;2:100015. Available from: https://dx.doi.org/10.1016/j. padiff.2020.100015.

14) Makinde OD. Free convection flow with thermal radiation and mass transfer past a moving vertical porous plate. International Communications in Heat and Mass Transfer. 1419;32(10):1411-1419. Available from: https://doi.org/10.1016/j.icheatmasstransfer.2005.07.005.

15) Ravikumar V, Raju MC, Chamkha AJ. Magnetic field effects on transient free convection flow through porous medium past an impulsively started vertical plate with fluctuating temperature and mass diffusion. International journal energy technology. 2013;5(4):1-8.

16) Ravikumar V, Raju MC, Raju GSS. Combined effects of heat absorption and MHD on convective Rivlin-Ericksen flow past a semi-infinite vertical porous plate with variable temperature and suction. Ain Shams Engineering Journal. 2014;5(3):867-875. Available from: https://dx.doi.org/10.1016/j.asej.2013. 12.014 .

17) Chamkha AJ. MHD flow of a uniformly stretched vertical permeable surface in the presence of heat generation/absorption and a chemical reaction. International Communications in Heat and Mass Transfer. 2003;30(3):413-422. Available from: https://dx.doi.org/10.1016/s0735-1933(03)00059-9.

18) Raju KVS, Reddy TS, Raju MC, Narayana PVS, Venkataramana S. MHD convective flow through porous medium in a horizontal channel with insulated and impermeable bottom wall in the presence of viscous dissipation and Joule heating. Ain Shams Engineering Journal. 2014;5(2):543-551. Available from: https://dx.doi.org/10.1016/j.asej.2013.10.007.

19) Siddulu MB, P PK, Lakshmi A, Matta S. Heat Transfer Reaction on a Viscous Dissipative Free Convective Radiating Stream over a Permeable Laminate within Presence of Induced Magnetic Field. International Journal of Innovative Technology and Exploring Engineering (IJITEE). 2020;9(3):2278-3075.

20) P PK, ShankarGoud B, SidduluMalga B. Finite element study of Soret number effects on MHD flow of Jeffrey fluid through a vertical permeable moving plate. Partial Differential Equations in Applied Mathematics. 2020;1:100005. Available from: https://doi.org/10.1016/j.padiff.2020.100005.

21) Poddar S, Islam MM, Ferdouse J, Alam MM. Characterstical analysis of MHD heat and mass transfer dissipative and radiating fluid flow with magnetic field induction and suction. SN Applied Sciences. 2021;3(4):1-17. Available from: https://doi.org/10.1007/s42452-021-04452-4.

22) Appidi L, Malga BS, Matta S, Kumar PP. Finite element analysis of viscous dissipation effects on unsteady free convention and mass transfer flow of fluid in a porous media with chemical reaction and heat source parameter. In: and others, editor. AIP Conference Proceedings. 2020. Available from: https://doi.org/10.1063/5.0014604.

23) Vijayaraghavan R, Karthikeyan S. Heat and Mass Transfer in Radiative Casson Fluid Flow Caused by a Vertical Plate with Variable Magnetic Field Effect. Advances in Physics Theories and Applications . 2017;67:6-23. Available from: 10.13140/RG.2.2.23014.06721.

24) Omamoke E, Amos E, Bunonyo KW. Radiation and Heat Source Effects on MHD Free Convection Flow over an Inclined Porous Plate in the Presence of Viscous Dissipation. American Journal of Applied Mathematics. 2020;8(4):190-206. Available from: https://dx.doi.org/10.11648/j.ajam.20200804.14.

25) Umamaheswar M, Raju MC, Varma SVK. Effects of Time Dependent Variable Temperature and Concentration Boundary Layer on MHD Free Convection Flow Past a Vertical Porous Plate in the Presence of Thermal Radiation and Chemical Reaction. International Journal of Applied and Computational Mathematics volume . 2017;3:679-692. Available from: https://doi.org/10.1007/s40819-015-0124-9. 